\title{
Movimientos mandibulares y posición de centricidad mandibular posutilización de un Jig Modificado de Lucia
}

Cristián Nilo, DDs 1 , Dagoberto Arcos, DDs 1 , Carolina Monsalve, DDs ${ }_{2}$, Raúl Frugone-Zambra*, MSc. y DHSc.

Recibido: 29 de marzo del 2016 Aprobado: 17 de agosto del 2016

*Autor de correspondencia: Raúl Frugone Zambra. Universidad de La Serena, La Serena, Chile. Brasil 798, Linares Chile. Teléfono: 5673 2211406. Correos electrónicos: raul@frugone.com y raulfrugone@gmail.com

Cómo citar este artículo: Nilo C, Arcos D, Monsalve C, Frugone-Zambra R. Movimientos mandibulares y posición de centricidad mandibular posutilización de un Jig Modificado de Lucia. Rev Nac Odontol. 2017;13(24):27-36. doi: http://dx.doi. org/10.16925/od.v12i24.1664

Resumen. Objetivos: con el fin de determinar si existen variaciones en la posición céntrica y en la dinámica condilar, en función de diferentes tiempos de utilización de un dispositivo oclusal anterior (Jig de Lucia modificado), se realizó un estudio pre-experimental. Métodos: el estudio consideró 15 sujetos sanos que fueron sometidos al uso de un Jig Modificado de Lucia (Jig). A cada uno se le registró el patrón céntrico, el movimiento condilar en sentido vertical y horizontal durante la apertura mandibular, así como el grado y patrón de apertura mandibular al inicio ( $\mathrm{T} 0$ ), a las 18 horas ( $\mathrm{T} 1$ ), a las 72 horas (т2), y a los siete días (т3). En la recolección de datos se utilizó un axiógrafo que utiliza ultrasonido (Arcus Digma ${ }^{\circ}$ ). Con el fin de contrastar la hipótesis nula se utilizó la dócima de McNemar y el análisis de Friedman. Resultados: los sujetos modificaron el tipo de registro de patrón céntrico de ambas articulaciones, variando de una forma de arco a una forma puntiforme, y el patrón de movimiento condilar en el plano horizontal. La máxima variación se observó en (т1). Conclusiones: al considerar el movimiento de rotación condilar en conjunto con la traslación en el plano horizontal, con el uso de un jig por 18 horas, en sujetos sanos y sin patología temporomandibular, se genera un patrón céntrico puntiforme, entendiéndose por esto que se genera un movimiento de arco dentro de un área de $1 \mathrm{~mm}^{2}$.

Palabras clave: centricidad mandibular, deprogramador anterior, jig, movimientos mandibulares, rotación condilar. 


\title{
Mandibular movements and mandibular centricity after using a Modified Lucia Jig
}

\begin{abstract}
Objectives: In order to determine if there are variations in the centric position and condylar dynamics, depending on different times of use of an anterior occlusal device (modified Lucia Jig), a pre-experimental study was conducted. Methods: The study included 15 healthy subjects who had to use of a Modified Lucia Jig (jig). The centric pattern, the condylar movement vertically and horizontally during mandibular opening, and the degree and pattern of mandibular opening was recorded for each participant at the beginning ( $\mathrm{T} 0$ ), after 18 hours ( $\mathrm{T} 1)$, after 72 hours ( 2 2), and after seven days ( $\mathrm{T} 3)$. In the data collection, an ultrasound-using axiograph (ARcus Digma ${ }^{\circledR}$ ) was employed. In order to test the null hypothesis, McNemar's decimals and Friedman's analysis were used. Results: Subjects modified the record type of the centric pattern for both joints, ranging from an arch shape to a punctiform shape, and the condylar movement pattern in the horizontal plane. The maximum variation was observed in ( $\mathrm{T} 1)$. Conclusions: When considering the condylar rotation movement together with the translation in the horizontal plane, with the use of a jig for 18 hours in healthy subjects without temporomandibular pathology, a centric punctiform pattern is generated, that is, an arc movement occurs within an area of $1 \mathrm{~mm}^{2}$.
\end{abstract}

Keywords: mandibular centricity, anterior deprogrammer, Jig, mandibular movements, condylar rotation.

\section{Movimentos mandibulares e posição de centricidade mandibular pós-utilização de um Jig de Lucia Modificado}

Resumo. Objetivos: com o objetivo de determinar se existem variações na posição cêntrica e na dinâmica condilar em função de diferentes tempos de utilização de um dispositivo oclusal anterior (Jig de Lucia Modificado), realizou-se um estudo pré-experimental. Métodos: o estudo considerou 15 indivíduos saudáveis que foram submetidos ao uso de um Jig de Lucia Modificado (jig). Cada um teve o padrão cêntrico e o movimento condilar em sentido vertical e horizontal durante a abertura mandibular registrados, bem como o grau e padrão de abertura mandibular ao início (T0), às 18 horas ( $\mathrm{T} 1)$, às 72 horas (т2), e aos sete dias ( $\mathrm{t} 3$ ). Na coleta de dados utilizou-se um axiógrafo que utiliza ultrassom (Arcus Digma $^{\circledR}$ ). Com o objetivo de contrastar a hipótese nula foi utilizado o teste de McNemar e o teste de Friedman. Resultados: os indivíduos modificaram o tipo de registro de padrão cêntrico de ambas as articulações, variando de uma forma de arco a uma forma puntiforme, e o padrão de movimento condilar no plano horizontal. A variação máxima foi observada em (T1). Conclusões: ao considerar o movimento de rotação condilar em conjunto com a translação no plano horizontal, com o uso de um jig por 18 horas, em indivíduos saudáveis e sem patologia temporomandibular, gera-se um padrão cêntrico puntiforme, entendendo-se assim que se gera um movimento de arco dentro de uma área de $1 \mathrm{~mm}^{2}$.

Palavras-chave: centricidade mandibular, desprogramador anterior, jig, movimentos mandibulares, rotação condilar. 


\section{Introducción}

Los aparatos interoclusales interpuestos entre los incisivos se han utilizado por mucho tiempo, con el fin de determinar y lograr una posición mandibular estable con respecto al cráneo. Dawson, en 1977, en la primera edición de Problemas Oclusales, señala: "La capacidad de manipular la mandíbula a través de un músculo contraído viene sólo con la práctica y el conocimiento" [1]. Sugiere cómo solo una vez se adquiera esa capacidad de obtener resultados coherentes, puede ser necesario recurrir a otros métodos, de manera que sea posible llevar el cóndilo a su posición terminal de bisagra, como, por ejemplo, al trabajar sobre la información propioceptiva generada por las interferencias oclusales artificiales que determinan patrones neuromusculares específicos. Con esto sería también posible guiar la mandíbula fácilmente a su posición.

Entre los métodos para lograr dicho objetivo, se encuentran la utilización del Jig de Lucia, de las láminas de Long o de los hilos de Roca, y de los planos de cobertura anterior, entre otros [2]. En un principio, esta técnica se indicó para la búsqueda de una posición mandibular gracias a la cual los cóndilos, al existir manipulación, tomaran la posición retruída ligamentosa (relación céntrica ligamentosa), posición que no ha demostrado ser funcional. Hoy en día, se trata de ubicar los cóndilos en una posición de relación céntrica con o sin disco articular interpuesto. En este sentido, se trata de obtener, en primer lugar, una posición de centricidad mandibular. Existen muchos métodos para ello, entre los cuales la posición inducida por manipulación resulta posterior, con relación a la obtenida a través de una acción funcional como lo es la deglución [3].

Al día de hoy, no existe una metodología única para obtener el registro de las relaciones intermaxilares universalmente aceptada; se utilizan diferentes técnicas con relativa equivalencia [4]. Un sistema estomatognático libre de patologías es una condición necesaria para la determinación correcta de la relación intermaxilar, por lo tanto, adquiere mayor relevancia en la selección de un método de registro, más que la elección de la modalidad del registro para un paciente dado. El tratamiento funcional debe primar al enfrentarse a esta situación [5].

Durante mucho tiempo ha sido cuestionado el concepto de relación céntrica y, de hecho, existen numerosas definiciones para ello. Sin embargo, antes de generar una definición se deben tener claras las características morfo-funcionales del sistema. El aparato dentario está sometido a una serie de cambios durante la vida y es por ello que se debe aplicar el concepto de equilibrio dinámico [6], en el cual la mandíbula posee una centricidad al momento de su rehabilitación y se encuentra en un equilibrio, tanto estático, como dinámico.

A pesar de toda la controversia, la relación céntrica sigue siendo un parámetro y punto de partida, es la referencia base en toda rehabilitación de la oclusión, aunque su definición conceptual aún está en constante modificación. Se acepta la posición más anterior, superior y medial del cóndilo en la cavidad glenoidea, definición que incluye la relación discal, o que no la incluye. En este caso, se podría hablar de relación céntrica adaptada [7].

A fin de registrar la relación céntrica existe la necesidad de "deprogramar" la neuromusculatura; se cree que los aparatos interincisales otorgan un engrama neuromuscular fisiológico, ya que se prescindiría de mecanismos propioceptivos protectores generados por algún contacto prematuro $[8$, 9]. Estos aparatos tienen la facultad de eliminar los contactos oclusales posteriores mientras están ubicados en la boca, así como al interferir en el trayecto de cierre mandibular hacia la máxima intercuspidación, generarían respuestas neurofisiológicas de tipo refleja. Los autores de este estudio, basados en los conceptos básicos de neurofisiología del movimiento, y en acuerdo con Pereira et al. [10], opinan que un engrama no puede ser borrado; lo que se lograría sería hipotéticamente una alteración de la información periférica y un estímulo a la acción del control central (sistema gamma eferente), lo que ayudaría a restaurar, en parte, y sin generar cambios plásticos, la simetría de acción de la musculatura y la estabilización de las posiciones y los movimientos mandibulares.

El proceso de la masticación es complejo y depende de múltiples arco reflejos. En este acto, la oclusión tiene importancia en la mantención de un movimiento armónico y preciso, es decir, para una dinámica estable. Esto significa que los movimientos deben partir de posiciones estables y, además, deben responder a patrones excéntricos también estables. Es así que se hace vital comprender el concepto de estabilidad mandibular asociado. Se define estabilidad mandibular como la facultad que tiene la mandíbula para mantenerse en equilibrio durante todo momento, en reposo o en movimiento, producto de la interacción de los distintos 
componentes que forman el sistema estomatognático y en ausencia de sintomatología [6]. Se desprende de esto que la mandíbula debe ser capaz de tener un equilibrio estable y un equilibrio indiferente, ayudada por la neuromusculatura, lo cual le permita tener esa capacidad de movilizarse y mantener la estabilidad en cada posición que adopte. Los elementos que se interponen entre los dientes ayudarían a determinar la posición de inicio de los movimientos, pero ¿son capaces de regularizar la armonía del movimiento mandibular?

Uno de los parámetros importantes durante el procedimiento de articulación de modelos es la localización el eje de bisagra. Con el propósito de registrar los movimientos mandibulares en los tres planos del espacio, se han desarrollado diferentes aparatos, uno de los cuales es el Gnathograph ${ }^{\circledast}$ [11].

Hoy en día, existen axiógrafos que permiten conocer y registrar la dinámica mandibular con base en la emisión y recepción de ultrasonido, situación que permite trabajar en tiempo real con la reproducción de los movimientos mandibulares del paciente. Además, estos axiógrafos permiten también un ajuste muy exacto del articulador, un análisis electrónico de posición y un registro del eje cinemático. El axiógrafo ARCUs Digma ${ }^{\circledR}$ es de los últimos sistemas de registro diseñados con el fin de determinar los movimientos mandibulares. Pröschel presentó en el 2002 un estudio para determinar la precisión de este axiógrafo sobre un articulador con valores predeterminados, y encontró una discrepancia no significativa de 1,5 grados. Sin embargo, presupone que sobre pacientes la variabilidad podría llegar a dos o tres grados de error [12].

Debido a la falta de evidencia existente en relación con la toma de registro interoclusal evaluado a través de instrumentos de última generación, el objetivo del presente estudio fue determinar si existe diferencia en la posición y trayectoria condilar durante los primeros milímetros de apertura mandibular, según el tiempo de utilización de un Jig Modificado de Lucia.

\section{Materiales y métodos}

Un total de 15 sujetos sanos, entre 18 y 25 años de edad (promedio $23 \pm 1,25$ ) de ambos sexos, fueron reclutados entre los alumnos de $3^{\circ}, 4^{\circ}$ y $5^{\circ}$ año de Odontología de la Facultad de Odontología de la Universidad del Desarrollo, en Concepción,
Chile. La recolección de datos se realizó durante un período de tres meses por un docente especialista en rehabilitación oral, capacitado en el uso del sistema ARcus Digma. Este estudio se realizó siguiendo la declaración de Helsinki. Todos los participantes fueron apropiadamente informados y firmaron un consentimiento a fin de confirmar que comprendieron completamente las características del estudio. En la recolección de datos, se cumplió con los protocolos sanitarios. Se excluyeron aquellos sujetos con patología muscular sistémica de base, patología temporomandibular según criterio diagnostico RDC/TMD, cefalea tensional y cervicalgia, sujetos sometidos a tratamiento de ortodoncia, sujetos con mordida invertida anterior, con overjet de más de $4 \mathrm{~mm}$, con mordida abierta anterior o mordida cruzada unilateral, con movilidad dentaria, con ausencia de piezas dentarias anteriores o desdentados totales.

Con el objetivo de evaluar si existen diferencias significativas en los registros de centricidad y movimientos condilares en sujetos antes de utilizar un Jig (т0), y después de utilizarlo por 18 h. (T1), 72 hrs. (т2), y siete días (т3), se realizó un estudio exploratorio con diseño pre-experimental. Se registraron las posiciones de centricidad neuromuscularmente guiada y las trayectorias condilares, utilizando el axiógrafo ARCus Digma (KaVoelektrotechnisches, werk, GMBF, Leutkirch, Germany).

Todos los estudiantes fueron invitados a participar. De 53 sujetos seleccionados según criterios, 15 de ellos se seleccionaron al azar a fin de conformar la muestra.

Cada sujeto fue sometido a un examen con el axiógrafo para generar los registros de cada evaluación ( $\mathrm{T} 0, \mathrm{~T} 1, \mathrm{~T} 2$ у т3). El sujeto fue sentado en posición ortostática mirando al horizonte. Se le secaron los dientes inferiores con aire comprimido, se ubicó la horquilla mandibular del instrumento en posición centrada sobre las caras vestibulares de ellos y se aplicó una resina acrílica (Structure ${ }^{\otimes}$ ) con el fin de fijarla. Posteriormente, se conectó el sistema al computador y se procedió a realizar la calibración individual del sistema para el paciente, procedimiento que se realizó cada vez que se pasó a generar los registros. Para ello, se solicitó al sujeto realizar movimientos de apertura y cierre mandibular no mayores a $10 \mathrm{~mm}$, y con adosamiento lingual al paladar para controlar el movimiento. A su vez, a cada uno de los sujetos se les proporcionaron las siguientes indicaciones sobre el uso del 
Jig: colocarse el Jig después del cepillado nocturno; dormir toda la noche con el Jig; cepillarse los dientes en la mañana sin Jig, sin generar contacto oclusal (con el dedo interpuesto entre los incisivos); y evitar tomar desayuno en el último día de uso del aparato hasta pasado el control, con el fin de no perder la respuesta neuromuscular obtenida durante el procedimiento.

Para el procedimiento de registro del movimiento de céntrica condilar (registro de patrón céntrico), se solicitó al sujeto realizar cuatro movimientos de rotación mandibular con adosamiento lingual. Tres de ellos fueron de entrenamiento y el cuarto se registró. Se definió como movimiento de rotación puntiforme un registro de movimiento dibujado en un área igual o menor a $1 \mathrm{~mm}^{2}$ (figura 1). Con el propósito de generar los registros de la trayectoria condilar en apertura y cierre, se pidió al sujeto realizar tres movimientos de máxima apertura voluntaria y cierre como entrenamiento previo, y luego se grabó el cuarto movimiento en el programa.

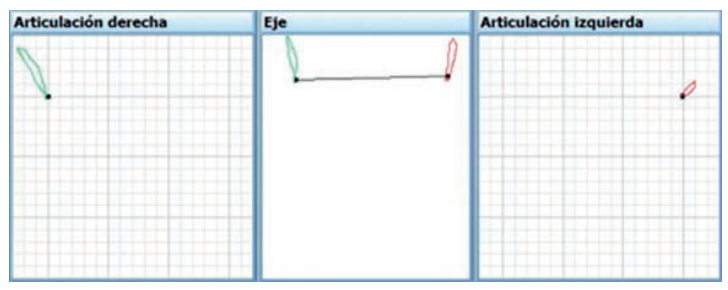

Figura 1. Se observa un patrón céntrico de la articulación derecha (en verde) en un área mayor de $1 \mathrm{~mm}^{2}$, y un patrón céntrico de la articulación izquierda (en rojo) en un área dentro de $1 \mathrm{~mm}^{2}$ (denominada como rotación puntiforme)

Fuente: elaboración propia

Una vez finalizado el proceso de registro inicial sin aparatos interoclusales instalados en la boca (т0), se confeccionó a cada sujeto un Jig de Modificado de Lucia (figura 2) [13]. El jig dejó 1 $\mathrm{mm}$ de separación entre los primeros dientes en contacto frente a una manipulación mandibular, y mantuvo una guía anterior generada por un punto de contacto de cada incisivo central inferior. Para la primera medición experimental, se solicitó al sujeto utilizar el jig durante $18 \mathrm{~h}$ sin removerlo. Posterior a esto, se realizó el mismo procedimiento de registro inicial. Para la segunda medición experimental, se solicitó al sujeto utilizar el jig por tres noches, así como no retirárselo al final de la última y acudir al control con el jig puesto. En esta sesión, se realizó la misma secuencia de registros iniciales con el ARCUS Digma ${ }^{\oplus}$. En la tercera medición experimental se solicitó al sujeto utilizar el jig por siete noches, sin retirárselo al final de la última, y acudir al control con el jig interpuesto.

Con el fin de contrastar la hipótesis nula, "no existe diferencia significativa en el registro de movimiento de céntrica condilar y en el registro de los movimientos condilares durante la apertura y cierre mandibular en sujetos antes de utilizar un Jig, y después de utilizarlo por $18 \mathrm{~h}, 72 \mathrm{~h}$, y por siete días", se utilizaron las décimas de McNemar y el análisis de Friedman.

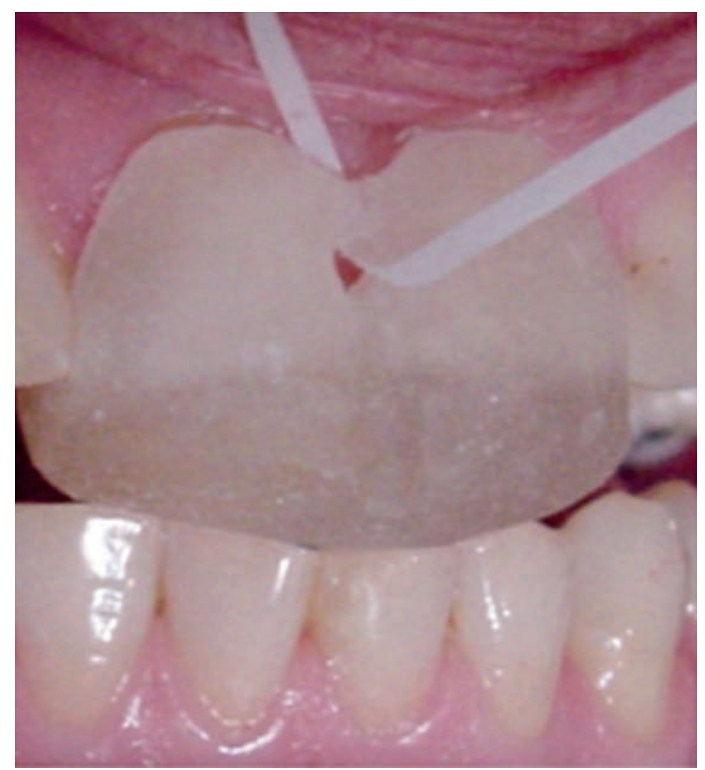

Figura 2. Jig puesto en boca con el paciente realizando un movimiento protrusivo

Fuente: elaboración propia

\section{Resultados}

De 15 individuos sanos a los que se sometió al uso de un jig con un tiempo máximo de siete días, se observó que el tipo de registro de patrón céntrico de ambas articulaciones en T1, T2 y T3 varió de una forma de arco a una forma puntiforme (tabla 1). 
Tabla 1. Datos generales para el patrón de céntrica

\begin{tabular}{|r|r|r|r|r|}
\hline & \multicolumn{2}{|c|}{ Derecha } & \multicolumn{2}{c|}{ Izquierda } \\
\hline & Arco (N) & Puntiforme (N) & Arco (N) & Puntiforme (N) \\
\hline T0 & 11 & 4 & 10 & 5 \\
\hline T1 & 4 & 11 & 3 & 12 \\
\hline T2 & 2 & 13 & 0 & 15 \\
\hline T3 & 2 & 13 & 0 & 15 \\
\hline
\end{tabular}

Fuente: elaboración propia

La distribución de la variable patrón del movimiento céntrico se expresó en tablas de contingencia que permitieron valorar la significancia estadística (tabla 2 y tabla 3 ).

Tabla 2. Tabla de contingencia de patrón céntrico derecho 18 h, 72 h, y 7 días (T1, T2, T3)

\begin{tabular}{|c|c|c|c|c|c|}
\hline \multicolumn{6}{|c|}{ Patrón céntrico $\mathrm{T} 1$} \\
\hline & & Puntiforme & Arco & Total & \\
\hline \multirow{3}{*}{$\begin{array}{l}\text { Patrón } \\
\text { céntrico } \\
\text { T0 }\end{array}$} & Puntiforme & 3 & 1 & 4 & \\
\hline & Arco & 8 & 3 & 11 & \\
\hline & Total & 11 & 4 & 15 & $\begin{array}{l}\mathrm{p} \text { value } \\
=0,039\end{array}$ \\
\hline \multicolumn{6}{|c|}{ Patrón céntrico $\mathrm{T} 2$} \\
\hline & & Puntiforme & Arco & Total & \\
\hline \multirow{3}{*}{$\begin{array}{l}\text { Patrón } \\
\text { céntrico } \\
\mathrm{T} 1\end{array}$} & Puntiforme & 11 & 0 & 11 & \\
\hline & Arco & 2 & 2 & 4 & \\
\hline & Total & 13 & 2 & 15 & $\begin{array}{l}\mathrm{p} \text { value } \\
=0,05\end{array}$ \\
\hline \multicolumn{6}{|c|}{ Patrón céntrico $\mathrm{T} 3$} \\
\hline & & Puntiforme & Arco & Total & \\
\hline \multirow{3}{*}{$\begin{array}{l}\text { Patrón } \\
\text { céntrico } \\
\text { T2 }\end{array}$} & Puntiforme & 12 & 1 & 13 & \\
\hline & Arco & 1 & 1 & 2 & \\
\hline & Total & 13 & 2 & 15 & $\begin{array}{l}\text { p value } \\
=0,039\end{array}$ \\
\hline
\end{tabular}

Fuente: elaboración propia
Tabla 3. Tabla de contingencia de patrón céntrico izquierdo $18 \mathrm{~h}, 72 \mathrm{~h}$ y siete días ( $\mathrm{T} 1, \mathrm{~T} 2, \mathrm{~T} 3)$

\begin{tabular}{|l|c|c|c|c|c|}
\hline \multicolumn{7}{|c|}{ Patrón céntrico T1 } \\
\hline & & Puntiforme & Arco & Total & \\
\hline $\begin{array}{l}\text { Patrón } \\
\text { céntrico } \\
\text { T0 }\end{array}$ & Puntiforme & 4 & 1 & 5 & \\
\hline & Arco & 8 & 2 & 10 & \\
\hline & Total & 12 & 3 & 15 & $\begin{array}{l}\text { p value } \\
=0,039\end{array}$ \\
\hline \multicolumn{7}{|c|}{ Patrón céntrico T2 } \\
\hline $\begin{array}{l}\text { Patrón } \\
\text { céntrico } \\
\text { T1 }\end{array}$ & Puntiforme & 12 & 0 & 12 & \\
\hline \multicolumn{7}{|c|}{ Puntiforme } & Arco & Total & \\
\hline & Arco & 3 & 0 & 3 & \\
\hline $\begin{array}{l}\text { Patrón } \\
\text { céntrico } \\
\text { T2 }\end{array}$ & Puntiforme & 15 & 0 & 15 & \\
\hline & Arco & 15 & 0 & 15 & p value \\
$=0,05$
\end{tabular}

Fuente: elaboración propia

En función de la apertura mandibular, se evaluó el trayecto condilar en el plano vertical y en el plano horizontal, así como el grado de apertura a nivel interincisal. Los valores promedios en el trayecto vertical condilar durante una apertura mandibular, tanto del lado derecho, como del izquierdo, para $\mathrm{T} 0, \mathrm{~T} 1, \mathrm{~T} 2$ y $\mathrm{T} 3$ se observan en la tabla 4 . Los valores promedios en el trayecto horizontal condilar durante una apertura mandibular, tanto del lado derecho, como del izquierdo, para $\mathrm{T} 0, \mathrm{~T} 1, \mathrm{~T} 2 \mathrm{y}$ т3, se observan en la tabla 5 .

Tabla 4. Promedios del movimiento vertical condilar en apertura mandibular

\begin{tabular}{|l|c|c|c|r|r|}
\hline & T0 & \multicolumn{1}{|c|}{ T1 } & \multicolumn{1}{c|}{ T2 } & \multicolumn{1}{c|}{ T3 } & p-value \\
\hline Derecho & 7,87 & 8,07 & 8,47 & 6,20 & 0,403 \\
\hline Izquierdo & 8,73 & 8,27 & 9,6 & 7,67 & 0,166 \\
\hline
\end{tabular}

Fuente: elaboración propia 
Tabla 5. Promedios del movimiento horizontal condilar en apertura mandibular

\begin{tabular}{|l|r|r|r|r|r|}
\hline & \multicolumn{1}{|c|}{ T0 } & \multicolumn{1}{c|}{ T1 } & \multicolumn{1}{c|}{ T2 } & \multicolumn{1}{c|}{ T3 } & p-value \\
\hline Derecho & 9,73 & 6,60 & 7,07 & 8,80 & 0,023 \\
\hline Izquierdo & 10,67 & 7,8 & 7,07 & 9,40 & 0,007 \\
\hline
\end{tabular}

Fuente: elaboración propia

El grado en el trayecto de apertura mandibular evidenció $31,27 \mathrm{~mm}$ de promedio en T0; 29,67 $\mathrm{mm}$ en $\mathrm{T} 1 ; 26,07 \mathrm{~mm}$ en $\mathrm{T} 2$; $\mathrm{y} 30,07 \mathrm{~mm}$ en $\mathrm{T} 3$ ( $\mathrm{p}=$ 0,312). El tipo de apertura se consideró como recto, desviado y desviado con corrección. Los resultados se observan en tablas de contingencia (tabla 6).

\section{Discusión}

De las cinco variables que se analizaron con el fin de determinar si existen cambios en los movimientos condilares mandibulares posterior al uso de un Jig, solo dieron una diferencia estadísticamente significativa el patrón de movimiento céntrico y el movimiento condilar mandibular horizontal.

El patrón de céntrica responde fundamentalmente al tipo de movimiento que realiza el cóndilo en la cavidad glenoidea en los primeros milímetros de apertura bucal. Este movimiento ha sido tema de discusión por décadas, en función de la existencia de rotación pura o de una roto-traslación que es característica en un eje de rotación helicoidal. De existir una rotación condilar pura durante los primeros milímetros del movimiento de apertura mandibular, dicho patrón debiera ser puntiforme. Sin embargo, la teoría del eje de rotación no puede explicar el movimiento puro, ya que el movimiento mandibular no ocurre alrededor de un eje fijo [14, 15]. Con el advenimiento del concepto del eje helicoidal que describe una roto-traslación del cóndilo durante todo momento, y basados en una anatomía funcional que no avala movimientos rígidos, se podría ratificar que dicho patrón de céntrica en todo momento muestra un trayecto condilar de arco [16]. Lo importante es que este patrón de arco, durante los primeros milímetros de apertura, se

Tabla 6. Tabla de contingencia patrón de apertura

\begin{tabular}{|l|l|r|r|r|r|r|}
\hline \multicolumn{7}{|c|}{ Patrón apertura T1 } \\
\hline
\end{tabular}


realizó en un área pequeña. Es por ello que en este estudio se consideró como marca puntiforme aquel registro de patrón céntrico que no ocupó más de $1 \mathrm{~mm}^{2}$, lo cual no significa que el movimiento de rotación se exprese realmente en un punto.

Entre todas las observaciones, ningún patrón de céntrica generó un registro $100 \%$ puntiforme. Sin embargo, la mayoría de los registros de ambas articulaciones, posterior al uso del dispositivo interoclusal, mostraron un arco dentro de $1 \mathrm{~mm}^{2}$. Esto se relaciona con los estudios que determinan cómo el movimiento condilar en posición céntrica, dentro de los primeros milímetros de apertura, no es solo de rotación, sino que contiene un componente de traslación. Además de las consideraciones sobre el eje helicoidal, se debe analizar el centro de rotación del movimiento mandibular. En carnívoros, se hipotetiza que este pasa a través de los cóndilos; no obstante, en primates y en omnívoros el centro rotacional no pasa por el cóndilo mandibular, debido a que el cóndilo se traslada durante el movimiento funcional. Ahora bien, un $23 \%$ de la apertura mandibular se realiza durante la traslación condilar, lo cual significa que la apertura de la boca se determina más por una rotación mandibular que por una traslación [17]. Se debe considerar que la rotación mandibular no significa rotación condilar, más aún, si se considera el concepto de centro instantáneo de rotación mandibular, el cual en omnívoros se ubicaría cerca del agujero mentoniano equidistante de ambos lados de la arcada [18].

Los datos generales que se observan en la tabla 1 demuestran que en la articulación derecha, 13 de 15 registros terminaron siendo puntiformes, y en la articulación izquierda todos los registros terminaron en la misma situación. A fin de verificar cómo han sido los cambios entre ambas situaciones de registro en el tiempo entre $\mathrm{T} 0$ у $\mathrm{T} 1, \mathrm{~T} 1$ у $\mathrm{T} 2$, у т2 у т3, se realizaron tablas de contingencia para cada una de las articulaciones. En la tabla de contingencia para la articulación derecha (tabla 2), se evidencia que de los cuatro patrones puntiformes en tiempo 0 , tres se mantuvieron como tales en $\mathrm{T} 1$ (McNemar 0,039<0,05), y se debe considerar que en este tiempo ya existían 12 registros de 15 en calidad de puntiforme. Entre T1 y T2, los 11 registros puntiformes se mantuvieron iguales (McNemar 0,5 $>0,05)$; у entre т2 у т3, de los 13 registros puntiformes de la articulación, 12 se mantuvieron como tales (McNemar $1>0,05)$. En la tabla de contingencia para la articulación izquierda (tabla 3), se evidencia que de los cinco patrones puntiformes en $\mathrm{T} 0$, cuatro se mantuvieron como tales en T1 (McNemar 0,039 < 0,05); y se debe considerar que en este tiempo ya existían 11 registros de 15 en calidad de puntiforme. Entre т1 y т2 los 12 registros puntiformes se mantuvieron como puntiformes (McNemar 0,25 > 0,05); y entre т2 y т3 los 15 registros puntiformes de la articulación izquierda se mantuvieron como tales (McNemar sin resultado debido a variables no dicotómicas). Frente a este análisis, se puede inferir que bastan $18 \mathrm{~h}$ de utilización de un Jig Modificado de Lucia para producir un cambio en la morfología del movimiento del patrón céntrico, y cómo el movimiento que ya es puntiforme, es decir, que se mantiene dentro de un área de $1 \mathrm{~mm}^{2}$, va a permanecer sin cambios. Por ende, para efectos de mantener o lograr un patrón de movimiento céntrico condilar dentro de un área de $1 \mathrm{~mm}^{2}$, no se justifica el uso de un dispositivo oclusal anterior por más de $18 \mathrm{~h}$.

Con respecto al desplazamiento condilar en sentido horizontal, también se encontraron diferencias estadísticamente significativas entre los tiempos de uso del jig. El movimiento condilar durante una apertura mandibular evidencia una mayor traslación que una rotación, y esta traslación posee un importante componente horizontal. Si la apertura mandibular se realiza con una gran traslación anterior en la coordenada $\mathrm{x}$, se verificará un movimiento mayor si se le compara con una apertura mandibular con poco componente anterior; dicho de otra manera, con un mayor componente rotacional. Al respecto, los resultados indican que existe diferencia significativa al analizar el componente horizontal en el eje $\mathrm{x}$ del movimiento condilar durante una máxima apertura (para el lado derecho $p=0,023$, y para lado izquierdo $p=0,007$; tabla 5). Resulta interesante observar que entre $\mathrm{T} 0$ y $\mathrm{T} 1$, y entre $\mathrm{T} 1$ y $\mathrm{T} 2$, los cambios en dicho movimiento resultan evidentes; sin embargo, dicha diferencia no es tan evidente si se compara $\mathrm{T} 0$ con $\mathrm{T} 3$.

Es probable que existan mecanismos neurofisiológicos de respuesta a la interferencia oclusal que se está provocando con el dispositivo anterior, los cuales podrían relacionarse, tanto a un patrón reflejo de tipo protector, como a una respuesta derivada de los cambios de longitud muscular que se provocan durante el uso de dicho aparato por cambio de dimensión vertical. Sin embargo, estas respuestas son transitorias, por lo que no sería raro volver a encontrar el mismo patrón de movimiento 
expresado en el desplazamiento horizontal a los siete días. La sensibilidad de los husos musculares se reduce frente a un aumento de la dimensión vertical [19]. También es posible que esta pueda ser efecto de un remodelado histoquímico de las fibras debido a dicha acción [20]. Esta plasticidad funcional de los husos del músculo masetero después de los cinco a 15 días, bajo un aumento de dimensión vertical inducido en ratas, dio paso a hipotetizar que la función del huso podría recuperarse en periodos mayores. Al respecto, Yabushita, Zeredo, Fujita, Toda y Soma demostraron que la función del huso muscular mostraba un alto grado de adaptabilidad [21]. Por ende, si hay adaptabilidad, podría ser factible que los movimientos mandibulares retomen su patrón habitual genéticamente determinado. Esto podría tener utilidad para ser analizado en función del manejo de patología muscular.

Para la obtención de un registro de centricidad mandibular, existen variados métodos que permiten determinar las diferentes posiciones mandibulares a las que se puede llegar. No obstante, ninguno utiliza una metodología que permita comparar posiciones reales condilares en un antes y un después, con instrumentos que den valores de medidas anatómicas utilizando un patrón oro como la resonancia magnética. En este estudio, no se compararon posiciones antes y después, debido a que una vez se retira el axiógrafo del ARCus Digma ${ }^{\circledR}$ en un paciente o en un sujeto, se pierde la calibración, de modo que solo se limitó a comprobar que el patrón céntrico tiende a ocupar un área menor a $1 \mathrm{~mm}^{2}$, condición que responde solo a uno de los parámetros fisiológicos de la centricidad mandibular. El otro parámetro es la posición de la centricidad condilar en la cavidad glenoidea, la cual debe ser evaluada imagenológicamente.

\section{Conclusiones}

Al considerar el movimiento de rotación condilar en conjunto con la traslación en el plano horizontal, en este estudio pre-experimental se encontró que la utilización de un Jig Modificado de Lucia en sujetos sanos y sin patología temporomandibular permite obtener un patrón de movimiento céntrico puntiforme, entendiéndose por ello que se genera un arco de movimiento dentro de un área de $1 \mathrm{~mm}^{2}$. Bastan 18 horas de utilización de un Jig Modificado de Lucia para producir un cambio en la morfología del movimiento del patrón céntrico. Se sugieren futuros estudios controlados con apoyo imagenológico para determinar la posición condilar.

\section{Referencias}

[1] Dawson P. Relación céntrica. En: Dawson P. Problemas oclusales. Argentina: Ed. Mundi; 1977. 69-93.

[2] Orozco A, Arroyo G, Martínez R, Ventura de la Torre J, Cañada D, Jiménez E. Relación céntrica: revisión de conceptos y técnicas para su registro. Parte II. Av Odontoestomatol. 2008; 24(6):369-76.

[3] Álvarez MC, Turbino ML, Barros C, Pagnano VO, Bezzon OL. Comparative study of intermaxillary relationships of manual and swallowing methods. Braz Dent J. 2009;20(1):78-83.

[4] Türp JC, Schindler HJ, Rodiger O, Smeekens S, Marinello CP. Vertikale und horizontale Kiefer-ralation in der rekonstruktiven Zahnmedizin. Eine kritische Literaturübersicht. Schweiz Monatsschr Zahmend. 2006;116:403-11.

[5] Palla S. Occlusal considerations in complete dentures. En: McNeill C (Hrsg.): Science and Practice of Occlusion. Chicago: Quintessence; 1997. 457-67.

[6] Frugone-Zambra R, Angulo M, Doñas P. Algunos conceptos biofísicos. En: Frugone-Zambra R, Angulo M, Doñas P. Atlas y manual práctico de preclínico para prótesis fija. Santiago: Editorial Texidó. 2002; 21-5.

[7] Pullinger A. Establishing better biological models to understand occlusion. I: TM joint anatomic relationships. J Oral Rehab. 2013; 40:296-318.

[8] Bodere C, Woda A. Effect of a jig on EMG activity in different orofacial pain conditions. Int J Prosthodont. 2008; 21(3):253-8.

[9] Wilson PH, Banerjee A. Recording the retruded contact position: a review of clinical techniques. $\mathrm{Br}$ Dent J. 2004; 196(7):395-402.

[10] Pereira M, Palinkas M, Hallak S, de Sousa L, Siéssere $\mathrm{S}$, et al. The effect of a Lucia jig for 30 minutes on neuromuscular re-programing, in normas subjects. Braz oral res. 2012; 26(6):530-5.

[11] Starcke EN. The history of articulators: From facebows to the Gnathograph, a brief history of early devices deveoped for recording condylar movement: Part II. J of Prosthod. 2002;11(1):53-62.

[12] Pröschel P, Morneburg P, Hugger A, Kordab B, Ottl $\mathrm{P}$, et al. Articulator-related registration. A simple concept for minimizing eccentric occlusal errors in the articulator. Int J Prosthodont. 2002;15:289-94. 
[13] Carrillo A. Evaluación electromiográfica del músculo temporal luego del uso del Jig de Lucia modificado por un intervalo de tiempo de 180 minutos en sujetos con normoclusión. Tesis. Colegio Odontológico del Perú. 1998. Disponible en: http://www. cop.org.pe/bib/tesis/ ANA\%20MARIA\%20CARRILLO\%20LIZARES.pdf

[14] Ferrario VF, Sforza C, Miani A Jr, Serrao G, Tartaglia G. Open-close movements in the human temporomandibular joint: does a pure rotation around the intercondylar hinge axis exist? J Oral Rehabil. 1996 Jun;23(6):401-8.

[15] Frugone R., Angulo M., Salinas R. Rotación condilar. Mitos y realidades. Revisión actualizada. Rev. Soc. Prot. Estom. de Chile. 1998;xiI(1).

[16] Airoldi G. Three dimensional description of mandibular finite helical axis pathways in asymptomatic subjects. Zürich: Medizinischen Fakultät der Universität Zürich;1994.
[17] Ferrario VF, Sforza C, Lovecchio N, Mian F. Quantification of translational and gliding components in human temporomandibular joint during mouth opening. Arch Oral Biol. 2005;50:507-15.

[18] Terhune C, Iriarte-Díaz J, Taylor A, Ross C. The insstantaneous center of rotation of the mandible in nonhuman primates. Integrative and Comparative Biology. 2012; 51(2):320-332.

[19] Yabushita T, Zeredo J, Toda K, Soma K. Role of occlusal vertical dimension in spindle function. J Dent Res. 2005;84(3):245-249.

[20] Santiwong P, Muramoto T, Soma K, Takano Y. Growth-associated protein-43 immunohistochemical and ultrastructural change in jaw muscle spindles of the rat following loss of occlusion. Arch Oral Biol. 2002;47:227-37.

[21] Yabushita T, Zeredo J, Fujita K, Toda K, Soma K. Functional adaptability of jaw-muscles spindles after bite-raising. J Dent Res. 2006;85(9):849-53. 